\title{
Comparative Analysis on Mobility Aware and Stateless Multicast Routing Protocols in Mobile Ad-Hoc Networks
}

\author{
Asst.Prof. A. Sofia Gunaseeli \\ Department of Computer Science and Engineering, Holy cross Engineering College, Tuticorin, India
}

\begin{abstract}
In this paper, the author has studied the performance of multicast routing protocols in wireless mobile ad-hoc networks. In MANET, for a protocol to be more efficient and high robust is a difficult task, due to the mobility of nodes and dynamic topology. It discovers the routing path by broadcasting message over the whole network, which results in considerable cost for routing discovery and maintenance. Moreover, the reliability of the discovered path can not be guaranteed, since the stabilities of nodes along such path are unpredictable. RBMR employs a mobility prediction based election process to construct a reliable backbone structure performing packet transmission, message broadcasting, routing discovery and maintenance. Another protocol novel Robust and Scalable Geographic Multicast Protocol (RSGM) is also analyzed. Several virtual architectures are used in the protocol without need of maintaining state information for more robust and scalable membership management and packet forwarding in the presence of high network dynamics due to unstable wireless channels and node movements.
\end{abstract}

Keywords: Multicast routing, geographic multicast, wireless networks, mobile ad hoc networks, geographic routing, scalable, robust, mobility prediction.

\section{Introduction}

A MANET is a self-organizing and self-configuring multi-hop wireless network, which can be instantly developed in situations where either a fixed infrastructure is unavailable (e.g., disaster recovery), or a fixed infrastructure is difficult to install (e.g., battlefields).In addition to the multi-hop nature of the wireless Ad-hoc networks and lack of a fixed infrastructure, these environments inherit the traditional problems of the wireless and mobile communications. Host mobility brings about a wide range of challenges in the design of the MANET protocols. In MANETs, to predict the mobility of a given host, the mobility parameters of the relative hosts also need to be taken into account, and so the mobility of such networks is generally hard to predict. Frequent and hard to predict topology changes due to the host mobility is the most important issue must be taken into consideration in mobile Ad-hoc networking. However, due to the limited radio propagation range of wireless devices, routes are often multi-hoped. Nodes in these networks move arbitrarily, thus network topology changes frequently especially in the high speed scenario.

Lots of popular multicast routing protocols, such as Adaptive Demand- Driven Multicast Routing and Multicast Ad hoc On-demand Distance Vector protocol, first build a multicast tree between a source and receivers and employ a process to detect broken link and repair the routes locally. These protocols perform well in terms of routing efficiency, however, they suffer from high mobility and highly dynamic topology. Other kinds of well known multicast routing protocols, such as On Demand Multicast Routing Protocol (ODMRP) [6], rely on periodic floods for route discovery and maintenance to ensure robustness against mobility and unreliable wireless link propagation. It, in fact, use more and more nodes as forwarding nodes when mobility increases, however, lead to trading off overhead for high packet delivery ratio to cope with mobility. In this paper, they propose a mobility-aware routing protocol; Reliable Backbone based Multicast Routing (RBMR), to improve the robustness and efficiency of multicast routing and delivery in high speed scenario. Different to other famous multicast protocols for MANETs, in RBMR, in order to achieve efficient and reliable packet forwarding, the most stable nodes are elected to be cluster leader forming a reliable backbone structure (RBS) to manage the content delivery, routing discovery and maintenance. Hence, in RBMR, routing discovery never suffers from the considerable cost aroused from flooding message over the whole ad hoc network either implicitly or explicitly.

Moreover, unlike previous works, a stability metric based upon mobility prediction is proposed to evaluate the reliability of nodes. Since the RBS is formed from these most relatively reliable nodes, the influence of nodes mobility is alleviated. Existing multicast protocols can take advantage of RBS by cooperating with it. They illustrate a routing discovery process based upon ODMRP. 
The remainder of this paper is organized as follows. In section II, we discuss the overview of multicasting protocols such as RBMR and RSGM with all its phases is illustrated. In section III, Analysis and results and finally in section IV, we conclude this paper.

\section{Reliable Backbone Based Multicast Routing}

In RBMR, three important things are discussed they are,

I. Link expiration time

Link expiration time indicates how reliable is the link between node $i$ and $j$.

The following formula calculates Link expiration time.

$$
\begin{aligned}
\operatorname{LET}(\mathrm{i}, \mathrm{j})= & \frac{-(a b+c d)+\sqrt{\left(a^{2}+c\right) r^{2}-(a d-b c)^{2}}}{a^{2}+c^{2}} \\
b & =x_{i}-x_{j} \\
d & =y_{i}-y_{j} \\
a & =v_{i} \cos \theta_{i}-v_{j} \cos \theta_{j} \\
c & =v_{i} \cos \theta_{i}-v_{j} \cos \theta_{j}
\end{aligned}
$$

II. Route expiration time:

$R E T(l)$-Route expiration time of the route path 1.

$$
\operatorname{RET}(l)=i \in(1,2, \ldots ., k)\left(\min T\left(l_{i}\right)\right)
$$

III. Node stability factor

NS(i)-stability level of the node i.

$$
N S(i)=\sum_{j \in N_{i}}(\operatorname{LET}(i, j))
$$

An example about node stability is depicted in figure 1. There are three links between node $A$ and its 1-hop neighbours: $\operatorname{LET}(A, C)=8, \operatorname{LET}(A, D)=3$ and $\operatorname{LET}(A, E)=2$. Hence, the node stability $\operatorname{NS}(A)=\operatorname{LET}(A, C)$ $+\operatorname{LET}(A, D)+\operatorname{LET}(A, E)=13$.

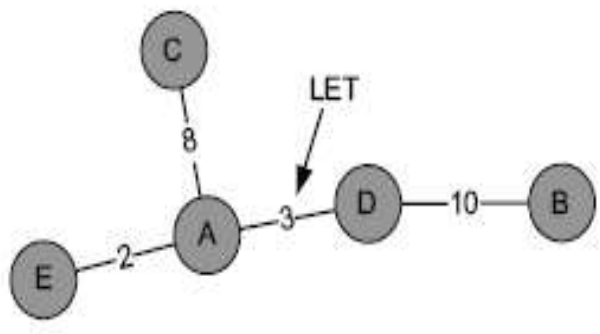

Fig1: Example of node stability [25]

\section{A. Mobility-aware cluster leader election}

In this mechanism, each node is either a cluster leader or a cluster member within a cluster after the process of cluster leader election. In RBMR, each cluster is formed from the 1-hop neighbourhood of the cluster leader. All the nodes elect the highest stable mobile node in their neighbourhood only by using 1-hop neighbourhood information. In order to react to changes of network topology, each node, e.g. node $j$, need to broadcast a 1-hop broadcast message, named CHELLO, periodically, e.g. every one second.

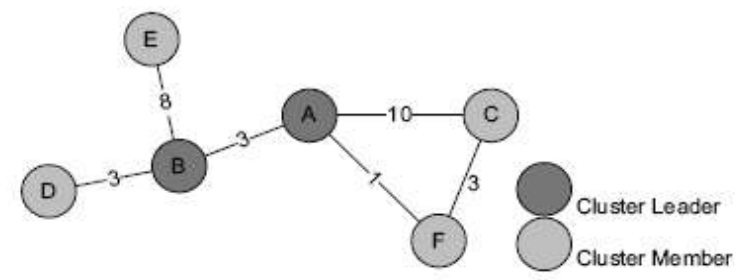

Fig 2: Example for leader election [25] 
In order to employ the motion information provided by GPS, as long as mobile node $j$ sends a CHELLO packet, its location, direction, and speed are appended to the packet. Hence, such message includes the fields $[N S(j), I D(j), C I D(j), x j, y j, v j, \theta j]$. All the 1-hop nodes receive CHELLO messages and update information of their neighbours for management. Once a 1-hop neighbour node, say mobile node $i$, received this message, it update the $N S(j)$ for node $j$ and the link reliability $L E T(i, j)$ between them is predicted by applying equation 1. Each mobile node also updates its own stability factor periodically by applying the equation 3 with the cycle of TStability. With all the node stability factors of all the nodes in the neighbourhood, nodes can easily elect a node in their leader, with the comparison metric in lexicographical ordering: $(N S(i), I D(i))$. In case two nodes in the neighbourhood take the same highest node stability, node prefers to elect the node with highest ID to be its cluster leader. Such nodes then transmits a CJOIN packet to its elected leader and update the CID (i) to be the ID of the leader.

\section{B. Mobility-aware gateway selection:}

A node is called a boundary node once it links to not only the nodes within the same cluster but also the nodes of the adjacent clusters. Note that a cluster header may also be a boundary node, since a cluster may have a link to a node which is also elected to be a leader by its neighbour or join another cluster leader with higher ${ }_{i}$ stability, $\mathrm{ID}_{i}$. There exist four different situations as following: 1) cluster leader directly link to cluster leader j. 2) member node in cluster i directly link to cluster leader j. 3) member node in cluster j directly link to cluster leader i. 4) member node in cluster i link to member node in cluster j. In order to achieve mobility-aware communication between two clusters and maintain a low overhead lever level, each cluster leader employs a detect process to discovery in an event driven manner.

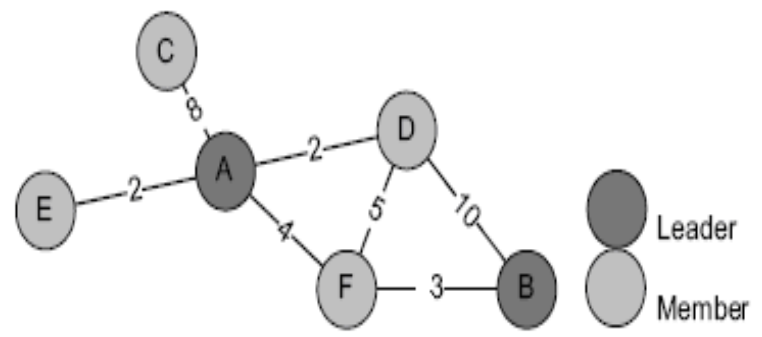

Fig 3: Example of routing path selection between two adjacent clusters. [25]

In this example, there exist four routing paths between leader $A$ and $B: I A D B, l A F B l A D F B$ and $I A F D B$. It's clearly that the gateway routing cannot be selected from both of $I A D F B$ and $I A F D B$ even $I A F D B$ take the highest route expiration time of 4, since both of them are 3-hops links. Among path $I A D B$ and path $l A F B$, the route expiration time of path $l A F B$ is higher than the one of path $l A D B$. As a result, path $l A F B$ become the routing path between leader $A$ and leader $B$. Eventually, after cluster leaders select their routing paths to the adjacent clusters, the RBS is formed.

\section{Multicast routing discovery}

Whenever a multicast source wishes to multicast data to the multicast group, it initiates the routing discovery process. Unlike the process in ODMRP, Join Query (JQ) messages only be broadcasted by RBS. Once the JQ message arrives at a multicast member, such member replies a Join Reply (JR) message. By the time the JR message arrives at a cluster leader, such leader become a forwarding node as well as its upstream cluster leader and the gateway nodes between them.

\section{Robust and Scalable Geographic Multicast Protocol}

A topology based multicast protocol generally has the following three inherent components that are difficult to scale they are

1 Group membership management

2 Creation and maintenance of a tree or mesh based multicast structure

3 Multicast packet forwarding

RSGM supports a two-tier membership management and forwarding structure.

At the lower tier, a zone structure is built. A leader manages the group membership and collects the positions of the member nodes in its zone. At the upper tier, the leaders of the member zones report the zone membership 
to the sources directly along a virtual reverse-tree-based structure.If a leader is unaware of the position or addresses of the source; it could obtain the information from the Source Home.

\section{A. Zone construction}

The Virtual zones are used as references for the nodes to find their zone positions in the network domain zone is set relative to a virtual origin located at (x0; y0).For simplicity, they assume all the zone IDs are positive. A zone ID will help locate a zone, and a packet destined to a zone will be forwarded toward its center.

\section{B. Group Membership Management}

The group membership is managed at two tiers. RSGM takes advantage of the virtual-zone-based structure to efficiently track the group membership and member positions.

- $\quad$ Local group membership management

- Membership management at the network level

\section{Source Initialization}

A multicast session $(\mathrm{G})$ is initiated and terminated by a source (S). To start a multicast session, $\mathrm{S}$ floods an ANNOUNCE (S, posS, groupIDs) message into the network (for reliability, promiscuous broadcasting is used in the flooding), where groupIDs are IDs of the groups ( $\mathrm{G}$ is one of them) for which $\mathrm{S}$ is the source. Upon receiving this message, a node $(\mathrm{N})$ interested in being the group member of $\mathrm{G}$ starts the joining process by unicasting to its zone leader a REFRESH message carrying the information of $\mathrm{S}$. After a session begins, $\mathrm{S}$ can piggyback its position (posS) to the multicast packets sent out to refresh its position at the receivers. When a member $\mathrm{M}$ moves to a new zone, the new leader can obtain the address and position of $\mathrm{S}$ from $\mathrm{M}$. To terminate $\mathrm{G}, \mathrm{S}$ floods an ANNOUNCE message with $\mathrm{G}$ removed from its group ID list.

\section{Source Tracking}

A source may move during the session time. The forwarders and receivers of the multicast packets can obtain the position of the source that is piggybacked with the packets.

To avoid a bottleneck, increase survivability, and improve transmission efficiency, the Source Home will not serve as the gateway for data traffic to the source.

The issues related to the management of Source Home are:

- Creation and maintenance of the Source Home with reliability, uniqueness, and consistency

- $\quad$ Efficient information update to the Source Home.

\section{Analysis And Results}

We evaluate the performance of RBMR by comparison with that of RSGM via packet-level simulations in various mobility scenarios. We evaluate the performance of RBMR and RSGM using the following metrics:

- $\quad$ Zone size Vs PDR

- $\quad$ Group size Vs PDR

- $\quad$ Zone size Vs Control Overhead

- $\quad$ Control Overhead Vs Max.Speed

- $\quad$ Network size Vs Control Overhead

- $\quad$ Group size Vs Control Overhead

\subsection{Packet Delivery Ratio:}

The ratio of the number of non duplicate data packets successfully delivered to the receivers versus the number of packets supposed to be received.

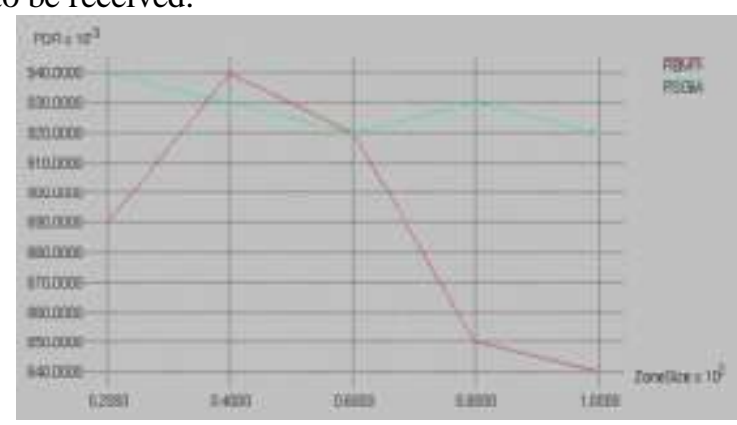

Fig 4. Zone size Vs PDR 
When the zone size decreases, packet delivery ratio increases.

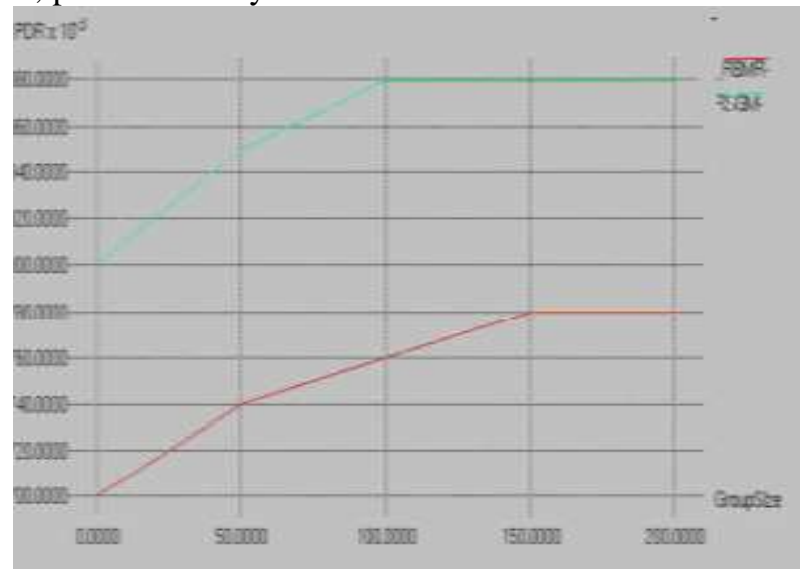

Fig 5. Group size Vs PDR

When the Group size increases the packet delivery ratio also increases. However RSGM has high packet delivery ratio in both cases.

\subsection{Control overhead}

It is the total number of control packets needed to establish a stable route from source to the multicast receivers.

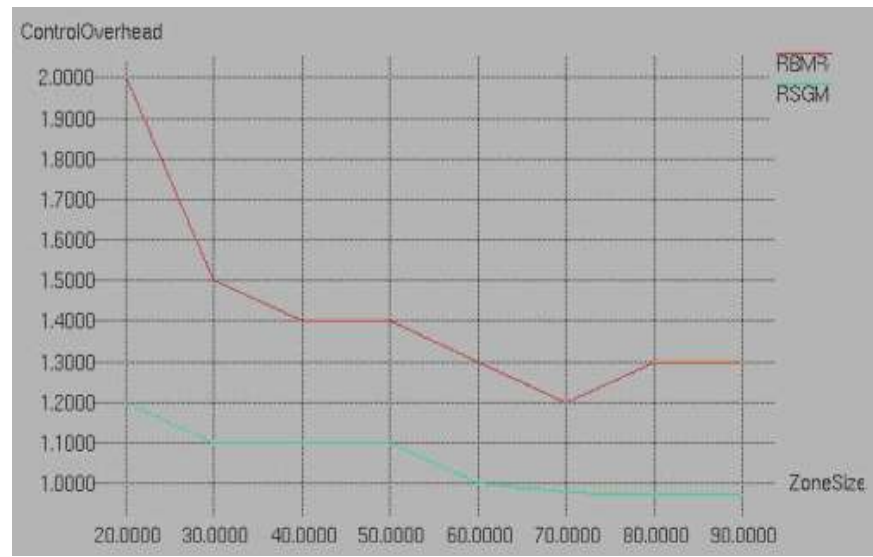

Fig 6. Zone size Vs Control overhead

When the zone size increases, control overhead decrease

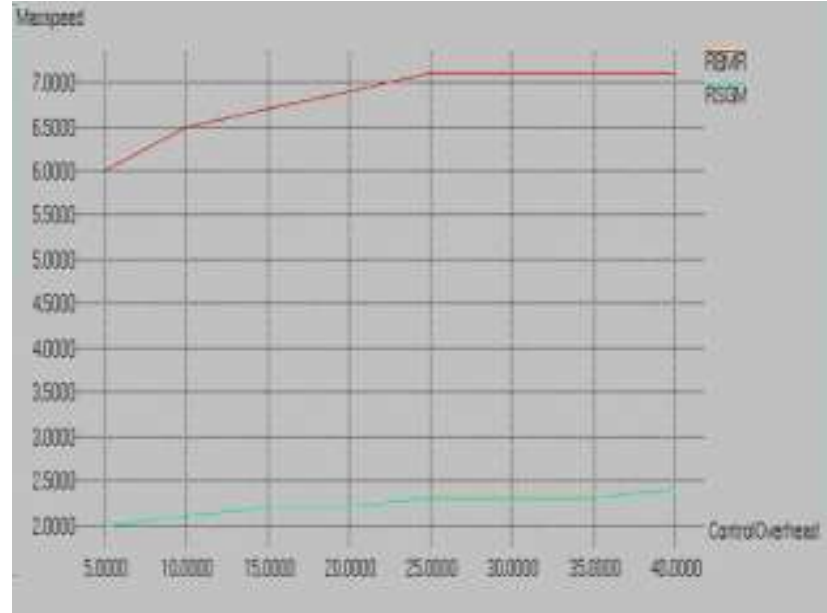

Fig 7. Control overhead Vs max.speed 
When the speed increases, overhead decreases.

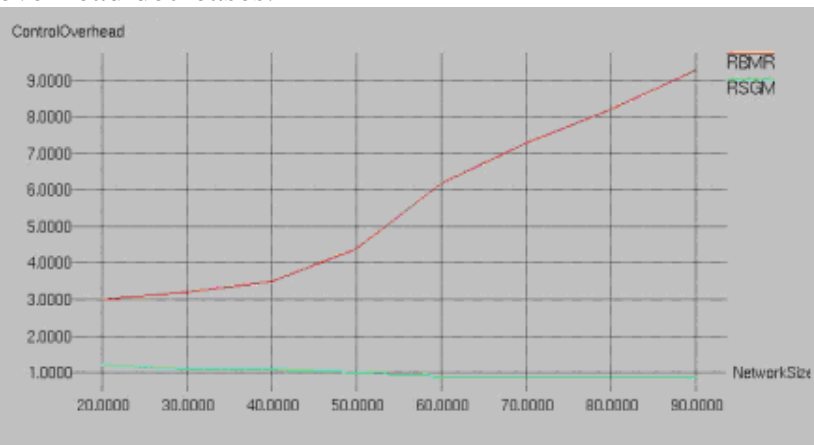

Fig 8. Network size Vs Control overhead

When the Network size increases, control overhead decreases.

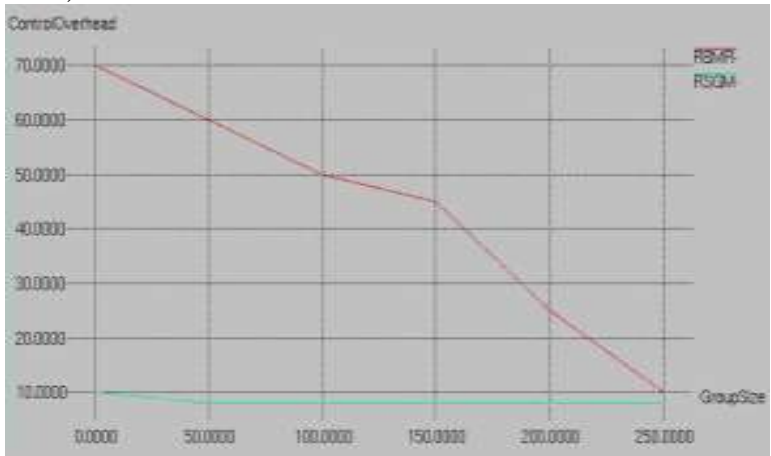

Fig 9. Group size Vs Control overhead

When the group size increases, control overhead decreases. However RSGM has low control overhead in all the cases.

\section{Conclusion And Future Work}

In previous sections, the author has studied the effectiveness and efficiency of protocols in multicast routing that have been proposed for ad-hoc networks. The proposed techniques preserve free mobility, multicast efficiency as well as robustness in wireless mobile Ad hoc networks and it also achieves good packet delivery ratio with low control overheads. The detailed simulator has enabled us to perform fair and accurate comparisons of the multicast protocols under a realistic wireless environment. Though the two protocols exhibit greater efficiency, from the comparison result it is clear that RSGM is more efficient than RBMR since it has higher packet delivery ratio and low control overhead.

\section{Acknowledgement}

The author would like to thank the anonymous reviewers for their many valuable comments and suggestions that helped to improve both the technical content and the presentation quality of this paper.

\section{References}

[1] L. Ji and M.S. Corson, "Differential Destination Multicast: A MANET Multicast Routing Protocol for Small Groups," Proc. IEEE INFOCOM '01, Apr. 2001.

[2] R. Beraldi and R. Baldoni, "A Caching Scheme for Routing in Mobile Ad Hoc Networks and Its Application to ZRP," IEEE Trans. Computers, vol. 52, no. 8, pp. 1051-1062, Aug. 2003.

[3] W.Su, S.Lee, and M.Gerla, "Mobility prediction in wireless networks." In MILCOM 2000. $21^{\text {st }}$ Centuary Military Communications Conference Proceedings, vol. 1, 2000.

[4] L.Bajaj, M.Takai, R.Ahuja, K.Tang, R.Bagrodia, and M.Gerla, "GloMoSim: A Scalable network simulation environment," UCLA Computer Science Dept Technical Report, vol. 990027, 1999.

[5] D. R. Johnson and D. A. Maltz, "Dynamic source routing in adhoc wireless networks," in Mobile Computing, (ed. T. Imielinski and H. Korth), Kluwer Academic Publishers, 19\%.

[6] S.Lee, W.Su, and M.Gerla, "On-demand multicast routing protocol in multi-hop wireless mobile networks," Mobile Networks and Applications, vol. 7, no. 6, pp. 441-453, 2002

[7] P.Sinha, R.Sivakumar, and V.Bhargavan, "MCEDAR: Multicast Core-Extraction Distributed Ad-hoc routing." In 1999 IEEE Wireless Communications and Networking Conferences, 1999, WCNC, 19999, pp 1313-1317

[8] J.Jetcheva and D.Johnson, “Adaptive demand driven multicast routing in multi-hop wireless ad-hoc networks," in proceedings of the $2^{\text {nd }}$ ACM international symposium on mobile ad-hoc networking and computing. ACM New york, NY, USA, 2001, pp. 33-44. 
[9] Y.Choi, B.Kim, K.Jung, H.Cho, and S.Kim, “ An Overlay Multicast mechanism using single-hop clustering and tree division for mobile ad-hoc networks," in IEEE 63 ${ }^{\text {rd }}$ Vehicular Technology Conference, 2006. VTC 2006-Spring, vol. 2, 2006.

[10] J.J. Garcia-Luna-Aceves and E. Madruga, “The Core-Assisted Mesh Protocol," IEEE J. on Selected Areas in Comm., vol. 17, no. 8, pp. 1380-1394, Aug. 1999.

[11] Gui and P. Mohapatra, "Scalable Multicasting for Mobile Ad Hoc Networks," Proc. IEEE INFOCOM, Mar. 2004.

[12] S. Wu and K.S. Candan, "GMP: Distributed Geographic Multicast Routing in Wireless Sensor Networks," Proc. 26th IEEE Int'l Conf. Distributed Computing Systems (ICDCS '06), 2006.

[13] S. Lee, W. Su, J. Hsu, M. Gerla, and R. Bagrodia, “A Performance Comparison Study of Ad Hoc Wireless Multicast Protocols,” Proc. IEEE INFOCOM, 2000

[14] Karp and H.T. Kung, "Greedy Perimeter Stateless Routing for Wireless Networks," Proc. MOBICOM, pp. 243-254, Aug. 2000.

[15] S-C.M. Woo and S. Singh, "Scalable Routing Protocol for Ad Hoc Networks," Wireless Networks, vol. 7, pp. 513-529, 2001.

[16] R. Bagrodia, R. Meyer, M. Takai, Y. Chen, X. Zeng, J. Martin, and H.Y.Song, .PARSEC: A Parallel Simulation Environment for Complex Systems,. IEEE Computer, vol. 31, no. 10, Oct. 1998, pp.77-85.

[17] T. Ballardie, P. Francis, and J. Crowcroft, .Core Based Trees (CBT) - An Architecture for Scalable Inter-Domain Multicast Routing, In Proceedings of ACM SIGCOMM'93, San Francisco, CA, Oct. 1993, pp. 85-95.

[18] Bommaiah, M. Liu, A. McAuley, and R. Talpade, .AMRoute: Adhoc Multicast Routing Protocol,. Internet-Draft, draft-talpade manetamroute- 00.txt, Aug. 1998, Work in progress.

[19] C. E. Perkins and E. M. Royer, “Adhoc on-demand distance vector (aodv) routing,” in Internet Draft draft-ietf-rnariet-aodv- 03.1 1,J une 1999.

[20] V. D. Park and M. S. Corson; "A highly adaptive distributed routing algorithm for mobile wireless networks," in Proceedings of 1997 IEEE Conference on Computer Communications, Apr 1997.

[21] R. Sivakumar, P. Sinha, arid V. Bharghavan, "Core extraction distributed ad hoc routing (cedar) specification," Internet Draft draft-ietfmanet-cedar-spec-OO.txtS,e pt. 1998. mobile ad-hoc wireless networks. In IEEE MILCOM 2001, pages 316-320.

[22] B. An and S. Papavassiliou. A mobility-based clustering approach to support mobility management and multicast routing in mobile adhoc networks. International Journal of Network Management, pages 387-395, 2001.

[23] G. Caizzone, P. Giacomazzi, L. Musumeci, and G. Verticale. Impact of user speed on the performance of a MAC protocol for vehicular adhoc networks. In 3rd Int. Conf. on Electronics, Hardware, Wireless and Optical Communications, WSEAS, Salzburg, 2004.

[24] P. Enge and P. Misra. Special issue on GPS: the Global Positioning System. Proc. of the IEEE, 87(1), January 1999, E. Royer and C. Perkins, "Multicast operation of the ad-hoc on demand distance vector routing protocol," in Proceedings of the $5^{\text {th }}$ annual ACM/IEEE international conference on Mobile computing and networking. ACM New York, NY, USA, 1999, pp. $207-218$.

[25] Yao Yu, Qi Zhang, and Sidan Du," Mobility Aware Multicast over MANET" 2009 ISECS International Colloquium on Computing, Communication, Control, and Management. 